\title{
Evaluation of Adaptive Boosting and Neural Network in Earthquake Damage Levels Detection
}

\author{
Mona Peyk-Herfeh \\ Science and Research Branch \\ Islamic Azad University, Qazvin, Iran
}

\author{
Asadollah Shahbahrami \\ Faculty of Engineering, \\ Department of Computer Engineering \\ University of Guilan, Rasht, Iran
}

\begin{abstract}
When an earthquake happens, the image-based techniques are influential tools for detection and classification of damaged buildings. Obtaining precise and exhaustive information about the condition and state of damaged buildings after an earthquake is basis of disaster management. Today's using satellite imageries has been becoming more significant data for disaster management. In this paper, an approach for detecting and classifying of damaged buildings using satellite imageries and digital map is proposed. In this approach after extracting buildings position from digital map, they are located in the pre- and post-event images. After generating features, genetic algorithm applied for obtaining optimal features. For classification, adaptive boosting and neural networks are utilized and compared with each other. These machine learning algorithms divided the damage levels into three classes of high, moderate and low levels. Experimental results which have been obtained from Bam earthquake images show that total accuracy of adaptive boosting for detecting and classifying of collapsed through uncollapsed buildings is about 79 percent while total accuracy of neural networks is about 65 percent.
\end{abstract}

\section{General Terms}

Classification, Image Processing, Adaptive Boosting, Neural Networks.

\section{Keywords}

Earthquake; Collapese Detection; Classification; Adaptive Boosting.

\section{INTRODUCTION}

Todays, a large number of massive earthquakes strike urban areas and huge number of fatalities and economic losses are reported. For instance, a strong earthquake of magnitude 6.5 struck the southeastern of Iran on December 26, 2003. The epicenter was located very close to the city of Bam. About 26000 people were killed and more than 30000 people were injured. When an earthquake happens, debris of buildings should be recorded for producing the damage map. In such destructive situation an accurate damage map from human settlements is very prominent and priceless for rescue team and disaster management and subsequently, arranges restoration activities [1]. Disaster management involves four phases: mitigation, preparedness, response and recovery. Mitigation phase takes place before or between disasters to decrease the influence of the events. This phase can be perceived as a part of recovery procedure if utilized after a disaster. Preparedness concentrates on improvement of community training, preparation for rescue forces and public knowledge. Response is the serious and critical phase happening after the disaster. This phase is described as the actions for saving human lives and preventing more damage in a disaster situation. Damage assessment, search and rescue are some actions that must be performed in response phase. Recovery phase consists of actions that are performed to restore the affected area to its pervious situation before disaster [2]. As it mentioned before, response phase is more prominent and critical in comparison the other phases. To better control of disaster management activities in this phase, reaching to an accurate damage map is important.

There are several strategies for calculating the damage map using remote sensing data. Selecting appropriate strategy according to the special situation can help disaster management to make significant decisions for saving people lives. Methodology of building damage assessment using remote sensing data can be divided into three categories. The first category belongs to the strategy that utilizes both pre- and post-event images. The main idea of this method is comparison between pre and post event images to determine the extent of earthquake damages. Some algorithms such as pixel-based and texture-based use this strategy [3]. Second category uses images that are taken after earthquake event because pre-earthquake images are not available always. Therefore, introducing a method that just uses only after image of earthquake for detecting the damages is valuable. But this method increases probability of error for finding the location of buildings and some buildings may not be evaluated [4]. The last category utilizes pre-, post-earthquake images and digital maps. This strategy extracts exact location of buildings. Buildings situation is inspected by computing spectral and textural features. Digital map provides information about the position of each building and this is the main advantage of this strategy and in comparison to the other previous strategies, it is more accurate and exhaustive [5]. Our approach belongs to this strategy.

After choosing appropriate strategy, selecting well-built classifier suited to classification application for obtaining an accurate damage map is noteworthy. The main challenging reasons to select neural networks versus adaptive boosting are the followings: first, many researchers have utilized neural networks for classification of earthquake damage [6-11]. Second, in compare to other classifications, neural networks have higher accuracy. Third, neural networks are nonlinear models, which makes them flexible in classifying real world such as earthquake damages [11]

Adaptive boosting is used here because it produces highly accurate classification rules by combining a number of weak hypothesis, each of them is only moderately accurate. The main benefit of adaptive boosting is that arbitrary classifier suitable for classification application can be chosen. Many researchers were used adaptive boosting for classifying applications such as change detection of buildings, roads and vegetation [12-13]. For realization of this purpose, remote sensing data and digital map are also used. In this approach, 
texture and color of roofs play an important role in classification because the roofs of damaged buildings have rougher texture in comparison with undamaged roofs. Our results show that the accuracy of adaptive boosting is about 79 percent while the accuracy of neural network is about 65 percent. This paper is an extension version of [14].

Compared to other related works, our main contributions are the followings:

- Calculating damage maps using pre- and postearthquake imagery of Quickbird satellite and vector maps in the ARC GIS and Matlab environment.

- In order to remove noise, some pre-processing techniques such as median filter are applied.

- Two type of classifier have been performed, adaptive boosting and neural network and then their accuracy is compared.

- Experimental results show that color and texture features play an important role compared to other features.

The reminder of this paper is organized as follows:

In the second section, some primary definitions and related works are described. In the third section, the proposed technique is expressed and experimental results are explained in the fourth section. The fifth part of this paper includes conclusions.

\section{BACKGROUND INFORMATION AND RELATED WORKS}

In this section, first the European micro seismic scale is described and then the steps of calculating damage map of earthquake are briefly explained. At the end, utilized classifiers and related works are investigated.

\subsection{European Micro Seismic Scale}

According to European micro seismic scale, earthquake damage can be divided into five classes that are shown in Table 1 [15].

In gardes of 1 and 2 only some cracks in walls, base and columns can be seen without any changing in texture of roofs.

Table 1. Classification of earthquake damage [15].

Classification of damage to building of reinforced concrete Grade 1: Negligible to slight damage

\begin{tabular}{l} 
Fine cracks in plaster over frame members or in walls at the \\
base. \\
Fine cracks in partitions and infill. \\
\hline Grade 2: Moderate damage \\
Cracks in columns and beams of frames and in structural \\
walls. \\
Cracks in partition and infill walls; fall of brittle cladding \\
and plaster. Falling mortar from the joints of wall panels. \\
\hline Grade 3: Substantial to heavy \\
Cracks in columns and beam column joints of frames at the \\
base and at joints of coupled walls. Spalling of concrete \\
cover, buckling of reinforced rods. \\
Large cracks in partition and infill walls, failure of \\
individual infill panels. \\
\hline Grade 4: Very heavy damage \\
Large cracks in structural elements with compression \\
failure of concrete and fracture of rebar; bond failure of \\
beam reinforced bars; tilting of columns. Collapse of a few \\
columns or of a single upper floor. \\
\hline Grade 5: destruction \\
Collapse of ground floor or parts (e. g. wings) of buildings. \\
\hline
\end{tabular}

This paper focuses on grades 3, 4 and 5. This is because textural changes of roofs are important and this kind of changes exists in these grades.

\subsection{Calculating Damage Map}

In order to detect vital changes in earthquake images, digital image processing techniques are applied in pre- and postevent images. The most common system that is used for calculating damage maps is shown in figure 1 [5-16-17]. The remote sensing technology plays an important role for detection of damaged levels. Satellite imageries like Quickbird imagery which it spatial resolution is about 2.4 meters in multispectral mode and 0.61 meters in panchromatic mode, acquire dependable data for producing damage maps. Therefore, for earthquake damage assessment, photo interpretation can be irreplaceable method that it depends on the resolution of the images. Eliminating the noise and geo-referencing of the images (is the process of placing an image into two dimensional spaces. In essence, geo-referencing pins a scanned map to particular geographical coordinates) are some of actions that are performed in the preprocessing step. In the next step the term of engine is used.

Indeed generating features, optimizing (genetic algorithm) and classification are performed in this step and the last step shows the output of system that is buildings damage map.

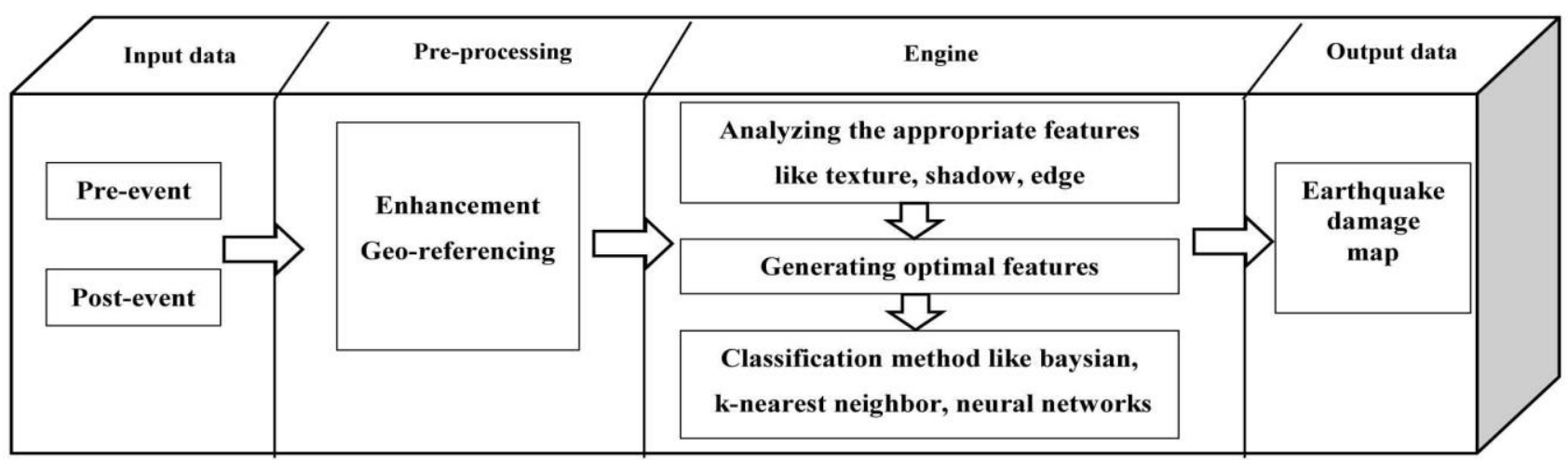

Fig. 1. The steps of calculating damage map of earthquake [5-16-17]. 


\subsection{Classification}

The texture of roofs changes, when an earthquake ruins a building. Building pixels can be classified by calculating texture features. Here, adaptive boosting and neural networks is used to divide pixels into three classes and total accuracy of them, is calculated. In the following, classifiers are described.

Adaptive boosting: Adaboost short for Adaptive boosting, is a meta-algorithm, and can be used in conjunction with many other learning algorithms to improve their performance [18]. Generally learning algorithms are either strong classifiers or weak classifiers. Strong classification algorithms use the techniques such as artificial neural network, support vector machine. Weak classification algorithms use the techniques such as decision trees, bayesian networks and random forests. The key idea of boosting is to create an accurate strong classifier by combining a set of weak classifiers. A weak classifier is only required to be better than chance, and thus can be very simple and computationally inexpensive. Adaptive boosting is adaptive because the instances misclassified by previous classifier are reorganized into the subsequent classifier. Ada-Boost is sensitive to noisy data and outliers. The boosting algorithm begins by assigning equal weight to all instances in the training data. It then calls the learning algorithm to form a classifier for this data, and reweighs each instance according to the classifier's output. The weight of correctly classified instances is decreased, and weight of misclassified instances is increased. This produces a set of easy instances with low weight, and a set of hard ones with high weight. In the next iteration, a classifier is built for the reweighed data, which consequently focuses on classifying the hard instances. Then the instances weights are increased or decreased according to the output of this new classifier. After all weights have been updated, they are renormalized so that their sum remains the same as it was before. After all iterations, the final hypothesis value is calculated.

The pseudo code for adaptive boosting algorithm is given as below:

- Input: a set $\mathrm{S}$, of ' $\mathrm{m}$ ' labeled examples: $\mathrm{S}=\left(\left(x_{i}, y_{i}\right), \mathrm{i}=\right.$ $(1,2, \ldots, \mathrm{m}))$, with labels in $\mathrm{Y}$.

- Learn (a learning algorithm)

- A constant L.

1. Initialize for all $\mathrm{i}: w_{j}(\mathrm{i})=1 / \mathrm{m} / /$ initialize the weights

2. for $\mathrm{j}=1$ to $\mathrm{L}$ do

3. for all $\mathrm{i}$ / $\quad / /$ compute normalized weights

$$
P_{j} \quad(\mathrm{i})=\frac{w(i)}{\sum_{i}^{m} w(i)}
$$

4. $h_{j}:=\operatorname{CART}\left(\mathrm{S}, p_{j}\right) \quad / /$ call weak Learn with Normalized weights

5. Calculate the error of $h_{j}$

$\varepsilon_{j}=\sum_{t} P_{j}(i)\left[h_{j}\left(x_{i}=y_{i}\right.\right.$

6. If $\varepsilon>\frac{1}{2}$ then

7. $\mathrm{L}=\mathrm{j}-1$

8. Go to 12

9. $\beta_{j}=\frac{\varepsilon_{j}}{1-\varepsilon_{j}}$

10. for all i:

$w_{j+1}(i)=w_{j}(i) \beta_{j}^{1-h_{j}\left(x_{i}=y_{i}\right)}$

// compute new weights

11. end for

12. output:

$h_{\text {final }}(x)=\sum_{j=1}^{L}\left(\log \frac{1}{\beta_{n}}\right)\left[h_{j}(x=y)\right]$
It is important to consider that the complexity of the strong classifier depends only on the weak classifiers. As weak classifier in this paper, a Classification And Regression Tree (CART) was used. The CART method produces binary decision trees distinguished by two branches for each decision node. CART recursively partitions the training data set into subsets with similar values for the target features. The CART algorithm grows the tree by conducting for each decision node, an exhaustive search of all available features and all possible splitting values; the optimal split is determined by applying well-defined criteria as Gini index or others ones [12].

Neural networks: This classification method is used extensively for classifying satellite imageries because they can be a calculation system instead of the non-linear functions. This system consists of many connected elements (neurons) that act together coordinately for solving a problem. A neural network for performing specific tasks, are set such as pattern recognition and information classification during the learning process. It is utilized extensively for detecting earthquake damage and produce damage map with high accuracy. Here, perceptron neural networks with two hidden layers by iteration of 1024 are utilized. Figure (3) shows the damage map created by neural networks that its accuracy is about 65 percent.

\subsection{Related Works}

For calculating earthquake map, lots of researches have been performed. Some of them have used different features such as shadow, edges and texture [19-23] and in some of them also have been applied machine learning approaches like neural networks, expert systems, support vector machine, K-nearest neighbors and baysian classification [3-16-24-25]. In 2010, a research has been performed using two categories of images, related to Bam and Kobe earthquakes. In this research machine learning approaches such as support vector machine, K-nearest neighbours and baysian classifier are utilized for calculating damage maps. The overall accuracy for each of classifiers was about $78 \%, 76 \%$ and $73 \%$ respectively [16]. In another research that was performed in 2011, the earthquake that happened in Italy was investigated. A kind of data fusion approach between SAR (Synthetic Aperture Radar) and optical data was presented. It has been shown that optical data are more suitable to distinguish between damage and non-damage classes, while SAR texture features allow to better distinguishing different classes of damages at block scale such as low and heavy damage [26]. Some researchers in 2008 investigated Bam earthquake. In this research, integration of high resolution satellite imageries and vector map has been proposed. In this method after extracting building positions from vector map and locating them to the both images of earthquake, different textural features measured and the building conditions are evaluated through a fuzzy interface system. Overall classification accuracy was about 74\% [5].

\section{PROPOSED APPROACH}

An approach is proposed for damage map generation using satellite imageries and digital map. Figure 2 depicts the proposed approach. As shown in the figure 2, digital map, both pre and post-event images of damaged area are utilized. This approach also uses adaptive boosting and neural networks as principal classifiers. Main steps of the proposed algorithm are described in figure 2 .

\subsection{Rational Equations}

At first, by utilizing rational equations can map the polygons on the post-event image for specifying the exact location of 
buildings because after an earthquake, the boundaries between the buildings are destroyed. The rational equations are shown in equation (1) and (2) [27].

$\mathrm{x}=\frac{p_{1}(x, y, z)}{p_{3}(x, y, z)}=\frac{\sum_{i=0}^{3} \sum_{j=0}^{i} \sum_{k=0}^{j} a_{m} X^{i-j} Y^{j-k} Z^{k}}{\sum_{i=0}^{3} \sum_{j=0}^{i} \sum_{k=0}^{j} c_{m} X^{i-j} Y^{j-k} Z^{k}}$

$\mathrm{y}=\frac{p_{2}(x, y, z)}{p_{4}(x, y, z)}=\frac{\sum_{i=0}^{3} \sum_{j=0}^{i} \sum_{k=0}^{j} b_{m} X^{i-j} Y^{j-k} Z^{k}}{\sum_{i=0}^{3} \sum_{j=0}^{i} \sum_{k=0}^{j} d_{m} X^{i-j} Y^{j-k} Z^{k}}$

In equations (1) and (2), $\mathrm{X}, \mathrm{Y}$ and $\mathrm{Z}$ are coordinate of ground map. Also $\mathrm{x}$ and $\mathrm{y}$ are coordinates in the image. $a_{m} \cdot b_{m}$ and $c_{m}$ are known as rational coefficients. In fact, by using rational equations can transfer the building polygons from 3D space (ground) to 2D space (image).

\subsection{Texture Analysis}

In this research, extracted buildings are evaluated using textural features. Textural features are calculated by applying filtering operation. A kernel with arbitrary size is moved on the image and a specific feature like mean is calculated and the value of feature is allocated to the central pixel. Here, textural features such as "1st Order Statistical", "Haralick", "Fractal" and "Variogram" have been selected. In the followings some of them are described.

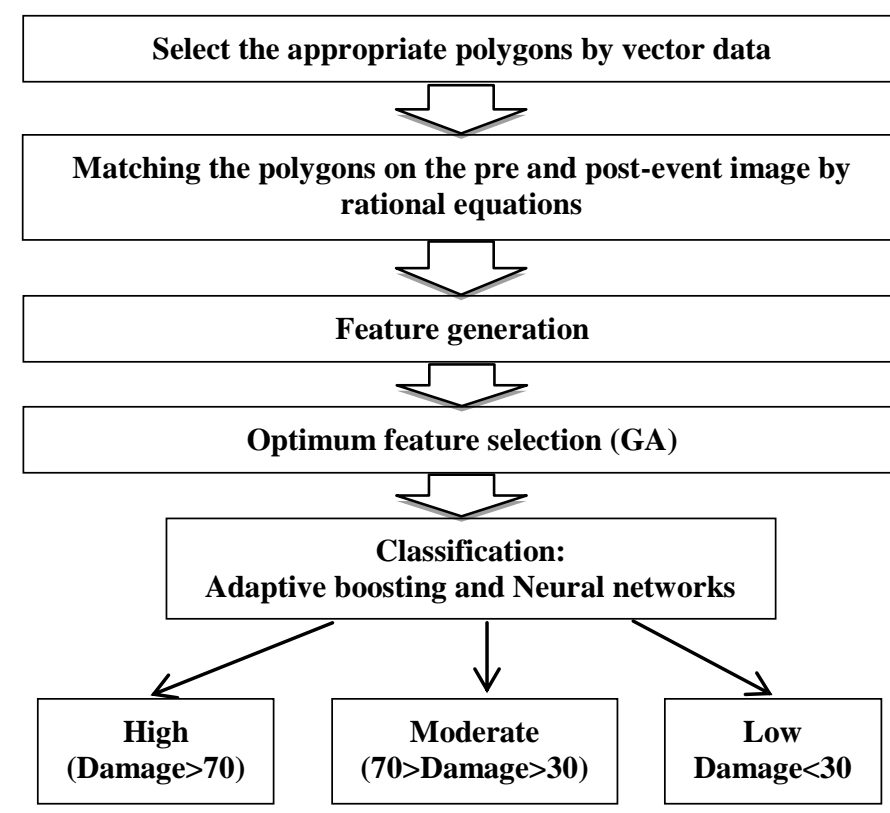

Fig. 2. The proposed algorithm.

First Statistical Features: In texture analysis, features such as mean, median, entropy, variance of gray value are utilized as 1st statistical textural feature. These features are generated from first order histogram $\mathrm{P}(\mathrm{I})$. In equation (3) $\mathrm{I}$ is the random variable which represents the grey levels.

$\mathrm{P}(\mathrm{I})=\frac{\text { number of pixels with grey level } \mathrm{I}}{\text { total number of pixels }}$

Second statistical features (Haralick Features): Grey Level Cooccorrence Matrices (GLCM) is one of the earliest methods for texture feature extraction [25]. Since then it has been widely used in many texture analysis applications and remained to be an important feature extraction method in the domain of texture analysis. Fourteen features were extracted by Haralick from the GLCM to characterize texture of image. Equation (4) shows the GLCM matrix [28-29].

$$
\begin{aligned}
& \operatorname{GLCM}_{d}^{\theta}= \\
& \frac{1}{R}\left[\begin{array}{ccccc}
\eta(0,0) & \eta(1,0) & \cdot & . & \eta\left(N_{g-1}\right) \\
\eta(1,0) & \eta(1,1) & \cdot & \cdot & \cdot \\
\cdot & \cdot & \cdot & \eta(i, j) & \cdot \\
\cdot & \cdot & \cdot & \cdot & \cdot \\
\eta\left(N_{g-1}, 0\right) & \cdot & \cdot & . & \eta\left(N_{g-1}, N_{g-1}\right)
\end{array}\right]
\end{aligned}
$$

$\eta(0,0)$ : The number of pixels with grey level $\mathrm{i}, \mathrm{j}$ in direction of $\theta$ and with distance of $\mathrm{d}$

$N_{g}$ : Number of grey levels

R: Total number of possible pairs

In this research, Haralick features on pre- and post images are calculated. The formulas of Haralick features are depicted in table 2 .

Semi-variogram Features: in this paper, semi-variogram features are also used. Table 3 shows the formula of these features.

Table 2. Some texture feature extracted from grey level co-occurance matrices [28].

\begin{tabular}{|c|c|}
\hline Features & Formulate \\
\hline Entropy & $-\sum_{i=0}^{M} \sum_{j=0}^{M} p(i, j) \mathrm{n}(p(i, j))$ \\
\hline Energy & $\sum_{i}^{M} \sum_{j}^{M} P^{2}[i, j]$ \\
\hline Contrast & $\sum_{i=0}^{M} \sum_{j=0}^{M}(i-j)^{2} p(i, j)$ \\
\hline Mean & $\frac{1}{2} \sum_{i}^{M} \sum_{j}^{M} i P[i, j]+j P[i, j]$ \\
\hline Variance & $\frac{1}{2} \sum_{i}^{M} \sum_{j}^{M}(i-\mu)^{2} P[i, j]+(j-\mu)^{2} P[i, j]$ \\
\hline Correlation & $\sum_{i=0}^{N_{g-1}} \sum_{j=0}^{N_{g-1}} \frac{\left(i-\mu_{i}\right)\left(i-\pi_{j}\right) p(i, j)}{\sigma_{j} \sigma_{i}}$ \\
\hline $\begin{array}{c}N_{g-1} N_{g-1} \\
\text { probability }\end{array}$ & $\sum_{i=0} \sum_{j=0}|i-j| p(i, j)$ \\
\hline Dissimilarity & $M_{i, j}{ }^{2}(P[i, j])$ \\
\hline
\end{tabular}

Table 3. Some texture features extracted from variogram [29].

\begin{tabular}{|c|c|}
\hline Feature & Formula \\
\hline $\begin{array}{c}\text { Sample } \\
\text { variogram }\end{array}$ & $\gamma_{k}(h)=\frac{1}{2 n h} \sum_{i=1}^{n(h)}\left\{D N_{k}\left(x_{i}\right)-D N_{k}\left(x_{i}+h\right)\right\}^{2}$ \\
\hline Madogram & $\gamma_{k}(h)=\frac{1}{2 n h} \sum_{i=1}^{n(h)}\left|D N_{k}\left(x_{i}\right)-D N_{k}\left(x_{i}+h\right)\right|^{2}$ \\
\hline Radogram & $\gamma_{k}(h)=\frac{1}{2 n h} \sum_{i=1}^{n(h)} \sqrt{\left|D N_{k}\left(x_{i}\right)-D N_{k}\left(x_{i}+h\right)\right|}$ \\
\hline
\end{tabular}




\begin{tabular}{|c|c|}
\hline $\begin{array}{c}\text { Cross } \\
\text { variogram }\end{array}$ & $\gamma_{k}(h)=\frac{1}{2 n h} \sum_{i=1}^{n(h)}\left\{D N_{j}\left(x_{i}\right)-D N_{j}\left(x_{i}+h\right)\right\}^{*}$ \\
& $\left\{D N_{k}\left(x_{i}\right)-D N_{k}\left(x_{i}+h\right)\right\}$ \\
\hline $\begin{array}{c}\text { Pseudo-cross } \\
\text { variogram }\end{array}$ & $\gamma_{k}(h)=\frac{1}{2 n h} \sum_{i=1}^{n(h)}\left\{D N_{j}\left(x_{i}\right)-D N_{k}\left(x_{i}+h\right)\right\}^{*}$ \\
& $\left\{D N_{j}\left(x_{i}\right)-D N_{k}\left(x_{i}+h\right)\right\}$ \\
\hline
\end{tabular}

Fractal Features: fractal geometry proposed by mandelbort in 1983. These features give a measure of the surface roughness. It means that when the fractal dimension is larger, then the texture is rougher. The mean and standard deviation of fractal dimension are calculated as textural features.

After generating the features, the genetic algorithm are used for optimum feature selection therefore the features that have less noise and dependency can be remained.

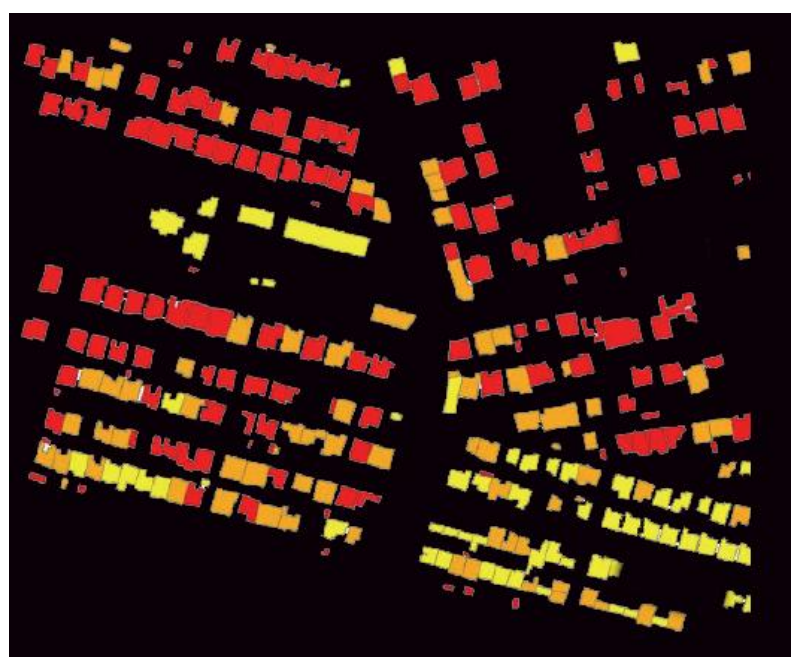

Fig. 3. Damage map created by neural networks.

\section{EXPERIMENTAL RESULT}

In this section our dataset, obtained results and discussion are proposed.

\subsection{Data Set}

In this paper, the proposed method is evaluated using pre- and post-event images of Bam earthquake. Figure 4 shows the data set that were utilized in this paper. Post-event image was taken approximately one week after earthquake and the preevent image was taken three months before earthquake.

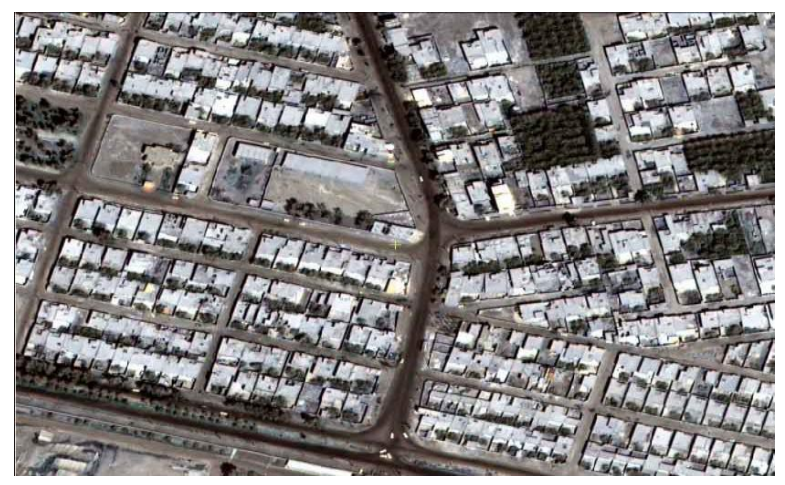

(a)

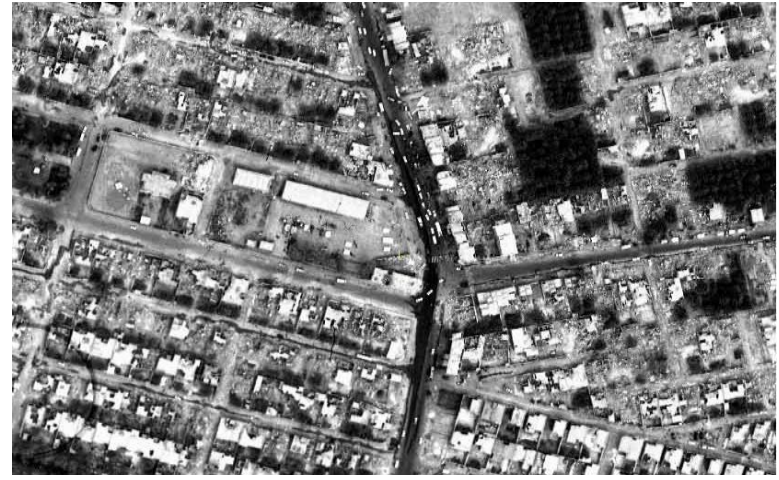

(b)

Fig. 4. Dataset. (a) pre-event image. (b) post-event image [30]

\subsection{Accuracy Assessment}

Mentioned classifiers (adaptive boosting and neural networks) are either right or wrong. Accuracy assessment tries to calculate how a classifier works the classification job well. These techniques are described as follows:

Overall accuracy: it is the total number of correctly classified samples divided by total number of samples. It measures the accuracy of the whole data without any indication of the accuracy of individual categories [16].

Producer's accuracy: it is the number of correctly classified samples of a specific category divided by the total number of reference sample for that class. It is an estimate of how many of buildings in each category are classified correctly [16].

User's accuracy: it is the number of correctly classified samples of a specific category divided by the total number of samples being classified as that category. It means, a user might wishes to know what proportion of buildings assigned to specific class, were correctly assigned [11].

\subsection{Results}

After performing the classification, it is important to evaluate the quality of the results. Overall accuracy, Producer's accuracy and User's accuracy are described in previous section.

Table 5 depicts the confusion matrices that are obtained by two classifiers adaptive boosting and neural networks. As are comprehended from this table, adaptive boosting is more accurate than neural networks in classifying of three levels of high, moderate and low. The number of buildings that are classified correctly by adaptive boosting are 132, 64 and 48 respectively. The numbers of correct classified buildings by neural networks are 109, 46 and 44 out of 309 . For example total number of misclassified buildings in high level damage for adaptive boosting is 32 while for neural network is 55 buildings. It means that 132 buildings were labeled correctly obtaining producer's and user's accuracy of 80.4 percent and 87.4 percent while by applying neural networks 109 buildings were labeled correctly and reaching to 66.4 percent and 83.2 percent producer's and user's accuracies. The overall accuracy is reached in this paper for adaptive boosting is about 79 percent and for neural networks about 65 percent.

The resulted damage map by applying adaptive boosting is shown in figure 5. As can be seen in this figure, red, orange and yellow colors depict high, moderate and low level of damage respectively. 


\section{CONCLUSIONS}

After geo-referencing the map and the pre- and post-event images, the exact location of buildings are extracted. Textural features have been calculated such as statistical, semi-variogram and fractal features. For the purpose of optimum feature selection, the genetic algorithm is applied. Adaptive boosting and neural networks have been utilized to divide damage into three classes of high, moderate and low level. Pre- and post-Quickbird satellite imageries of Bam earthquake were used to detect changes and to provide buildings damage map. Confusion matrix and accuracy assessment techniques that are obtained from mentioned classifiers showed stimulating difference in their performance. In the other words, implementation of adaptive boosting is more complex than neural networks and in addition, adaptive boosting is more computationally intensive than neural networks. Overall accuracy of adaptive boosting was about 79 percent that is more accurate than neural networks which its accuracy was about 65 percent in same condition.

\section{REFRENCES}

[1] Chini, M. N. Pierdicca, and W. J. Emery. 2009. "Exploiting SAR and VHR Optical Images to Quantify Damage Caused by the 2003 Bam Earthquake." IEEE Transactions on Geoscience and Remote Sensing. 47: 145-152.

[2] Rezaeian, M. 2010. "Assessment of Earthquake Damages by Image-Based Techniques." PhD Thesis. ETH Zurich.

[3] Cheema, U. 2007 "Expert Systems for Earthquake Damage Assessment." IEEE Aerospace and Electronic Systems Magazine. 22: 6-10.

[4] Saito, K., and R. Spence. 2005. "Rapid Damage Mapping using Post-Earthquake Satellite Images." International Symposium on Geoscience and Remote Sensing. 4: $2272-2275$

[5] Samadzadegan, F., and H. Rastiveisi, 2008. "Automatic Detection of Damaged Buildings, using High Resolution Satellite Imagery and Vector Data." the International Archives of the Photogrammetry, Remote Sensing and Spatial Information Sciences. 37: 415-420.

[6] Ito, Y., M. Hosokawa, H. Lee, and J. G. Liu. 2000. "Extraction of Damaged Regions using SAR Data and Neural Network." International Archives of Photogrammetry and Remote Sensing. 33: 156-163.

[7] Yah, L. H., Y. J. Yan, and J. S. Jiang. 2003. "VibrationBased Damage Detection for Composite Structure using
Wavelet Transform and Neural Network Identification.' Composite Structure. 60: 403-412.

[8] Bakhary, N., H. Hao, and A. J. Deeks. "Damage Detection using Artificial Neural Network with Consideration of Uncertainties." Engineering Structure. .29: 2806-2815.

[9] Hung, S. L., C. Y. Kao. 2002. "Structural Damage Detection using the Optimal Weights of the Approximating Artificial Neural Networks.” Earthquake Engineering and Structural Dynamics. 31: 217-234.

[10] Gonzalez, M. P., and J. L. Zapico. 2008. "Seismic Damage Identification in Buildings using Neural Networks and Modal Data." Computers and Structures. 86: 416-426.

[11] Zhang. G. P. 2000. "Neural Networks for Classification a Survay." IEEE Transaction on Systems. 30: 451-462.

[12] Gonulalan, C. 2010. "An AdaBoost Based Approach to Automatic Classification and Detection of Buildings Footprints, Vegetation Areas and Roads from Satellite Images." MSc Thesis. Northeastern University. Boston.

[13] Manchini, A., E. Frontoni, and P. Zingaretti. 2009. "Automatic Extraction of urban Objects from MultiSource Aerial Data." International Arshives of Photogrammetry and Remote Sensing. 38: 13-18.

[14] Herfeh, M. P., Shahbahrami, A., Miandehi, F. P., 2013, "Detecting Earthquake Damage Levels using Adaptive Boosting." $8^{\text {th }}$ Iranian Conference on Machine Vision and Image Processing. pp:251-256.

[15] Grunthal, G. "European Micro Seismic Scale. 1998 "European Center for Geodynamics and Seismology.

[16] Rezaeian, M. 2012. "Automatic Classification of Collapsed Buildings using Stereo Arial Images." Internatinal Journal of Computer Application. 46: 35-42.

[17] Kouchi, K., and F. Yamazaki. 2005. "Damage Detection Based on Object-based Segmentation and Classification from High-resolution Satellite Images for the 2003 Boumerdes, Algeria Earthquake.” In: Proceeding Asian Conference on Remote Sensing. pp:7-11.

[18] Freund, Y., and R. E. Schapire, 1996. "Experiments with a New Boosting Algorithm." In Machine Learning: Proceedings of the Thirteenth International Conference. 148-156.

Table 4. Accuracy assessment of classifiers

\begin{tabular}{|c|c|c|c|c|c|c|}
\cline { 2 - 7 } \multicolumn{1}{c|}{} & \multicolumn{4}{c|}{ Visual interpretation } \\
\hline & \multicolumn{2}{|c|}{ High damage=164 } & Moderate damage=78 & \multicolumn{2}{c|}{ Low levels of damage=67 } \\
\cline { 2 - 7 } & Adaptive \\
Confusion matrix & $\begin{array}{c}\text { Neural } \\
\text { betworks }\end{array}$ & $\begin{array}{c}\text { Adaptive } \\
\text { boosting }\end{array}$ & $\begin{array}{c}\text { Neural } \\
\text { networks }\end{array}$ & $\begin{array}{c}\text { Adaptive } \\
\text { boosting }\end{array}$ & $\begin{array}{c}\text { Neural } \\
\text { networks }\end{array}$ \\
\hline High damage & 132 & 109 & 12 & 14 & 7 & 8 \\
\hline Moderate damage & 19 & 35 & 64 & 46 & 12 & 15 \\
\hline Low levels of damage & 13 & 20 & 2 & 18 & 48 & 44 \\
\hline
\end{tabular}




\begin{tabular}{|c|c|c|c|c|c|c|}
\hline \multirow{2}{*}{ Accuracy assessment } & \multicolumn{3}{|c|}{ Adaptive boosting } & \multicolumn{3}{c|}{ Neural networks } \\
\hline Overall & \multicolumn{3}{|c|}{$78.9 \%$} & & $64.4 \%$ \\
\hline Producer's & $80.4 \%$ & $82 \%$ & $71.6 \%$ & $66.4 \%$ & $58.9 \%$ & $65.6 \%$ \\
\hline User's & $87.4 \%$ & $67.3 \%$ & $76.1 \%$ & $83.2 \%$ & $47.9 \%$ & $53.6 \%$ \\
\hline
\end{tabular}

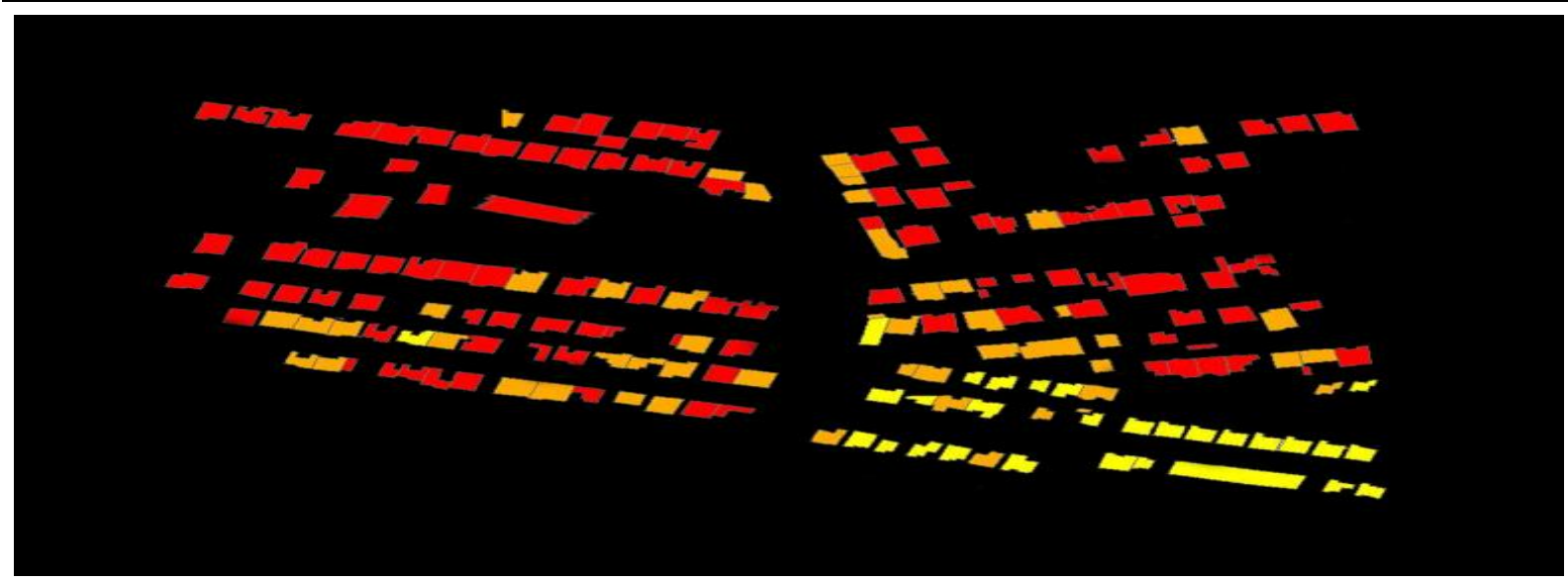

[19] Turker, M., and B.T. San. 2004. "Detection of Collapsed Buildings Caused by the 1999 Izmit, Turkey Earthquake Through Digital Analysis of Post-Event Aerial Photographs." International Journal of Remote Sensing. 25: 4701-4714.

[20] Vu, T. T., M. Matsuoka, and F. Yamazaki. 2004. "Shadow Analysis in Assisting Damage Detection Due to Earthquakes from Quickbird Imagery." International Archives of Photogrammetry Remote Sensing and Spatial Information Sciences, 607-610.

[21] Turker, M., and E. Sumer, 2008. "Building-Based Damage Detection due to Earthquake using the Watershed Segmentation of Post-Event Aerial Images." International Journal of Remote Sensing. 29: 3073-3089.

[22] Li, P. 2011. "Urban Building Damage Detection from Very High-Resolution Imagery by One-Class SVM and Shadow Information.” IEEE International Symposium on Geoscience and Remote Sensing. 1409 - 1412.

[23] Sumer, E., and M. Turker. 2005. "Building Damage Detection from Post-Earthquake Aerial Imagery using Building Grey-Value and Gradient Orientation Analyses." Proceedings of $2^{\text {nd }}$ International Conference on Recent Advances in Space Technologies. 577-582.

[24] Rehor, M., and T. Vögtle. 2008. "Improvement of Building Damage Detection and Classification Based on
Laser Scanning Data by Integrating Spectral Information." International Archives of Photogrammetry and Remote Sensing, 1599-1605.

[25] Ahadzadeh, S., M. Valadanzouj, S. Sadeghian and S. Ahmadi. "Detection of Damaged Buildings After an Earthquake using Artificial Neural Network Algorithm.' International Archives of the Photogrammetry, Remote Sensing and Spatial Information Sciences. 37: 369-371.

[26] Dell'Acqua, F. 2011. "Earthquake Damages Rapid Mapping by Satellite Remote Sensing Data: L'Aquila April 6th, 2009 Event.” IEEE Journal of Selected Topics in Applied Earth Observation and Remote Sensing. 4: 935-943.

[27] Demir, A., E. Sertel, N. Musaoglo, and C. Ormeci. 2008. "Accuracy Assessment of Radargrammetric DEMs Derived from Radarsat-2 Ultrafine Mode.” International Archives of Photogrammetry and Remote Sensing. 38.

[28] Haralick, R. M., 1979. "Statistical and Structural Approaches to Texture.” 67: 786-804.

[29] Gonzalez, R. C., and R. E. Woods. 2007. "Digital Image Processing." Prentice Hall $3^{\text {rd }}$ edition.

[30] Kiavarz Moghadam, M., and F. Samadzadegan. 2008 "Post-Earthquake Building Damage Assessment using High-Resolution Satellite Imageries in the Case of the 2003 Bam, Iran, Earthquake.” Geo Physical Reseach. 